\title{
Prolonged extracorporeal circulation
}

\author{
THORKILD FREDERIKSEN, J ØRGEN ROSEN, INGE H. RYGG, \\ ERNA CHRISTENSEN, AND FREDERIK THERKELSEN \\ From the Departments of Thoracic Surgery $R$ and Anaesthesia, Rigshospitalet, Copenhagen, Denmark
}

Interest in prolonged perfusions has increased pari passu with the possibility of treating the more complicated cardiac deformities. Prolonged perfusions may be of interest also in functional disturbances arising during operations using the heart-lung machine, since it may be difficult to discontinue the perfusion before cardiac function is again satisfactory. Furthermore, the biological changes which take place during prolonged perfusion reflect, in an intensified degree, the changes that occur during an ordinary brief perfusion (Andersen, 1961). Even though the outcome is fatal after such a long-lasting perfusion, it is of interest to investigate the biochemical changes during the perfusion, especially if the patient has not succumbed to the perfusion. It is of even greater interest to ascertain whether the patient might have survived such a long-lasting perfusion had he not succumbed to other causes. A very prolonged perfusion in a patient who has survived has not yet been reported. This is not surprising, since prolonged extracorporeal circulation has usually been necessitated by a life-threatening condition. The data from a 12-hour perfusion are presented.

\section{CASE REPORT}

E. D. O., a boy aged 6 years 10 months, case record $402 / 61-62$, had been cyanosed from birth, and the cyanosis had been gradually increasing. He had never been able to play.

On admission he showed physical retardation, intense cyanosis, pronounced clubbing of the fingers, and thick and curved nails. There was no hepatomegaly nor splenomegaly. Angiocardiography in 1959 (Fig. 1) had revealed transposition of the great vessels and a ventricular septal defect. Chest radiography showed enlargement of the left heart to $10 / 17 \cdot 5 / 3 \cdot 5 \mathrm{~cm}$. There was great prominence of the pulmonary arch and the lung markings were increased. Blood pressure was $95 / 60 \mathrm{~mm}$. $\mathrm{Hg}$, haemoglobin $14.7 \mathrm{~g} . / 100 \mathrm{ml}$, , and sedimentation rate 0 . The boy was $106 \mathrm{~cm}$. in height and weighed $16.7 \mathrm{~kg}$. Pre-operative investigation of the capillary blood revealed standard bicarbonate $16.1 \mathrm{mEq} / 1$. plasma, base excess $-10.3 \mathrm{mEq} / 1$. blood, $p \mathrm{H} \mathrm{7.27,} \mathrm{PCO}_{2} 43 \mathrm{~mm}$. $\mathrm{Hg}$, and oxygen saturation $50 \%$. The urine contained no albumin. Serum creatinine was 0.9 and serum protein $7.6 \mathrm{mg} . / 100 \mathrm{ml}$. An electrocardiogram showed right axis deviation and tall, pointed P2 and P3. The patient was severely ill, and radical surgery was undertaken.

OPERATION AND EXTRACORPOREAL CIRCULATION ON 9 February 1962 trans-sternal thoracotomy correcting the transposition was performed under general anaesthesia. After premedication with atropine the anaesthesia was induced by a quick-acting barbituric acid (Narcodorm = enibomal sodium NFN) intravenously. Before intubation suxamethonium chloride was administered. Anaesthesia was maintained by nitrous oxide-halothane-flaxedil.

During the extracorporeal circulation small supplementary doses of Narcodorm were given. An Engström respirator was used for the ventilation.

The correction was performed through a right-sided ventriculotomy, the pulmonary artery and the root of

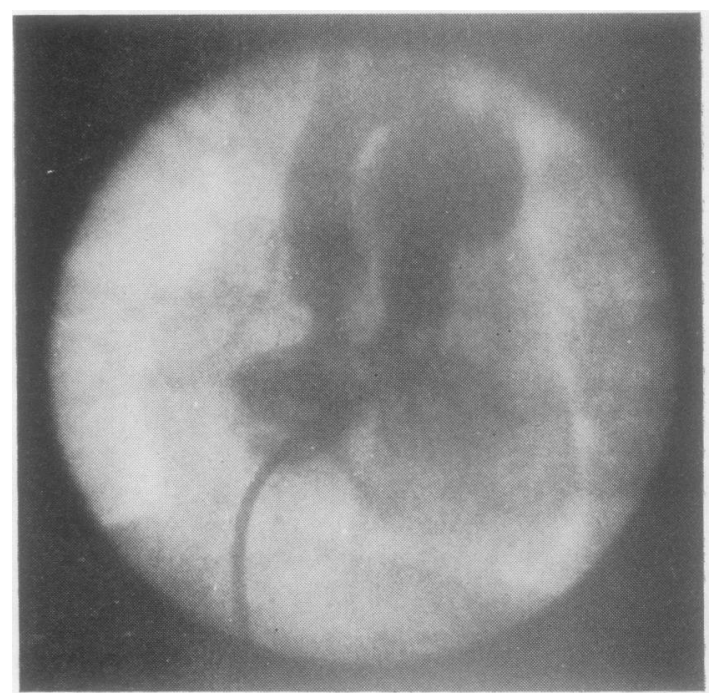

FIG. 1. Angiocardiography 1959. Contrast medium was injected into the right ventricle, passing into the aorta through a ventricular septal defect, into the left ventricle, and thence into the pulmonary artery. 
the aorta being mobilized and the pulmonary artery pulled forward in front of the aorta. The base of the aorta was then displaced to the left and a prosthesis sutured to the ventricular septal defect.

During the cardiotomy extracorporeal circulation was maintained by the Rygg-Kyvsgaard heart-lung machine type IV (Arnfred, Rygg, Frederiksen, Engell, Poulsen, and Rosen, 1961 ; Frederiksen, Rygg and Therkelsen, 1962), and general hypothermia was maintained by means of a heat exchanger. The temperature was brought down to about $23^{\circ} \mathrm{C}$. and the heating was started when the correcting prosthesis had been fixed. At $32^{\circ} \mathrm{C}$. spontaneous ventricular tachycardia occurred, but the rhythm could be slowed down by the administration of Lidocaine. Then it was found that a total block was present. An attempt to discontinue the extracorporeal circulation resulted in a high pressure in the right ventricle and pulmonary artery. At one time a pressure of $90 \mathrm{~mm}$. $\mathrm{Hg}$ was found in the pulmonary artery as compared with $75 \mathrm{~mm}$. $\mathrm{Hg}$ in the aorta. There was a question of pulmonary hypertension, and the right ventricle failed after a short time despite stimulation by calcium chloride and drugs of the adrenaline group. It also proved impossible to keep the right ventricle going by a pace-maker, and there was uninterrupted, rather coarse fibrillation in the form of large undulating movements. In the hope of improving the right ventricle thus damaged by anoxia, the extracorporeal circulation was continued, draining from the right and left atrium. Fig. 2 shows the perfusion data.

The temperature curve showed a period of hypothermia of about two hours and thereafter an almost constant temperature of $36^{\circ} \mathrm{C}$. From the flow curve it is evident that a high flow was needed in order to keep the blood pressure above $60 \mathrm{~mm}$. Hg. During long periods the flow was $4.4 \mathrm{l} . / \mathrm{min}$., corresponding to $6.3 \mathrm{l} . / \mathrm{m} .{ }^{2} / \mathrm{min}$. (body surface $0.7 \mathrm{m.} .^{2}$ ). It may be seen also that the flow diminished in proportion to the hypothermia. The cause of the high flow was possibly aortic insufficiency with recirculation through the left-sided sucker. During a short period the flow curve is interrupted. This represents the time when an attempt was made to discontinue the extracorporeal circulation. During this period the pump was turned on and off at a highly varied frequency, with a resulting variation in the flow. After this the flow was fairly constant throughout the remaining period of the perfusion. Most of the time the arterial pressure curve showed values below $100 \mathrm{~mm}$. Hg. During the period that we tried to discontinue the perfusion the pressure was fluctuating. The venous pressure was most of the time between 10 and $20 \mathrm{~mm}$. $\mathrm{Hg}$.

Fig. 3 gives the biochemical data from the extracorporeal circulation. From the start of the perfusion the oxygen saturation was brought up to almost $100 \%$ where it remained throughout the operation. The standard bicarbonate and base excess values increased as the oxygen saturation rose and remained constant during the primary perfusion. After the cardiotomy, owing to the interrupted perfusion and the poor circulation, anaerobic metabolic products presumably accumulated in the blood, making it acid. Some time after the perfusion was re-started in full the acidity of the blood f values only to increase again during the last hours of the extracorporeal circulation, indicating that the flow had not been sufficient throughout the entire vascular bed. These variations in the acid-base status are reflected in the $p \mathrm{H}$ values of the blood.

The near-normal $\mathrm{PCO}_{2}$ was obtained by administering $2 \% \mathrm{CO}_{2}$ in pure oxygen to the oxygenator.

An electrocardiogram revealed the above-mentioned changes of ventricular tachycardia and total block passing into constant, coarse ventricular fibrillation.

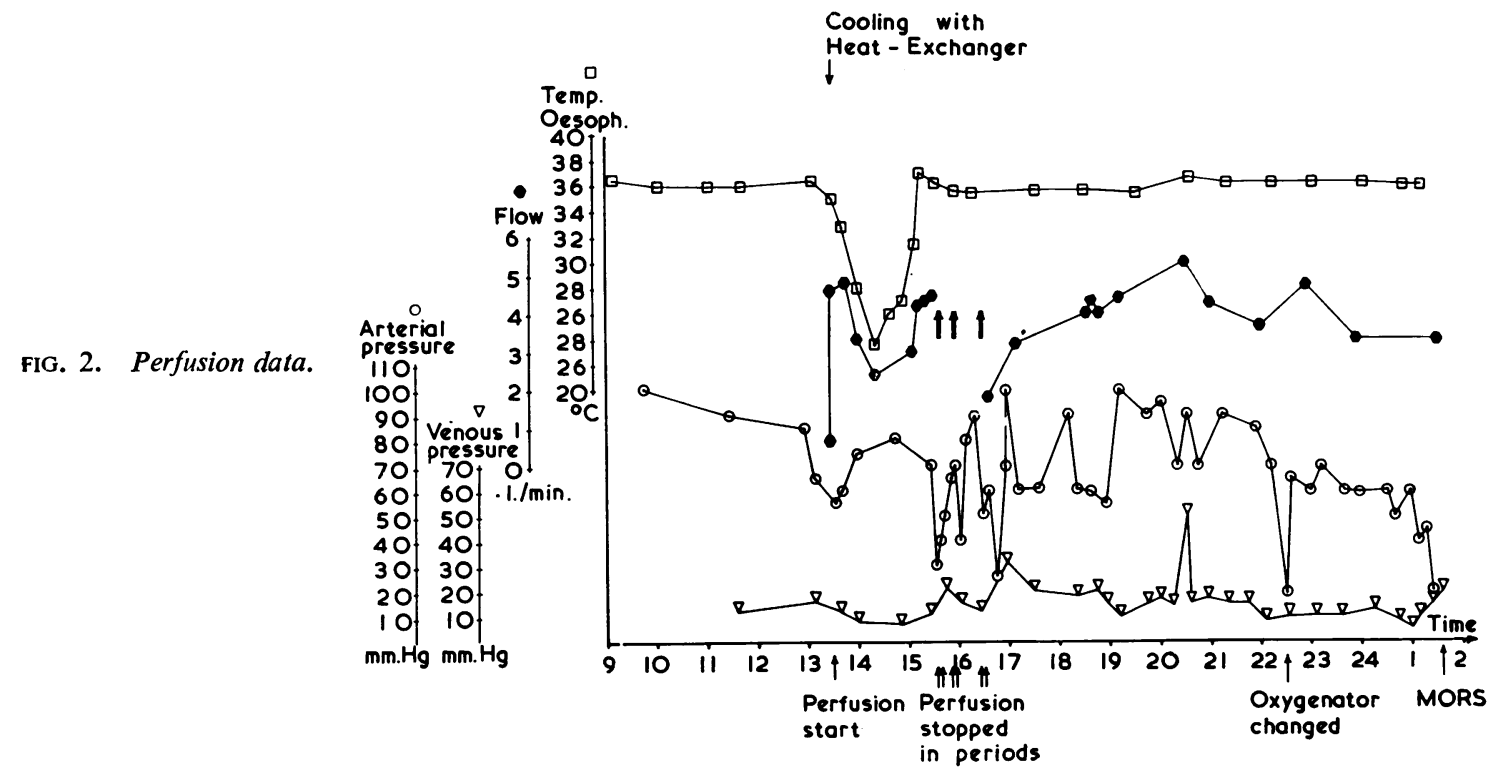

Cooling with

Heat - Exchanger

$\downarrow$ 


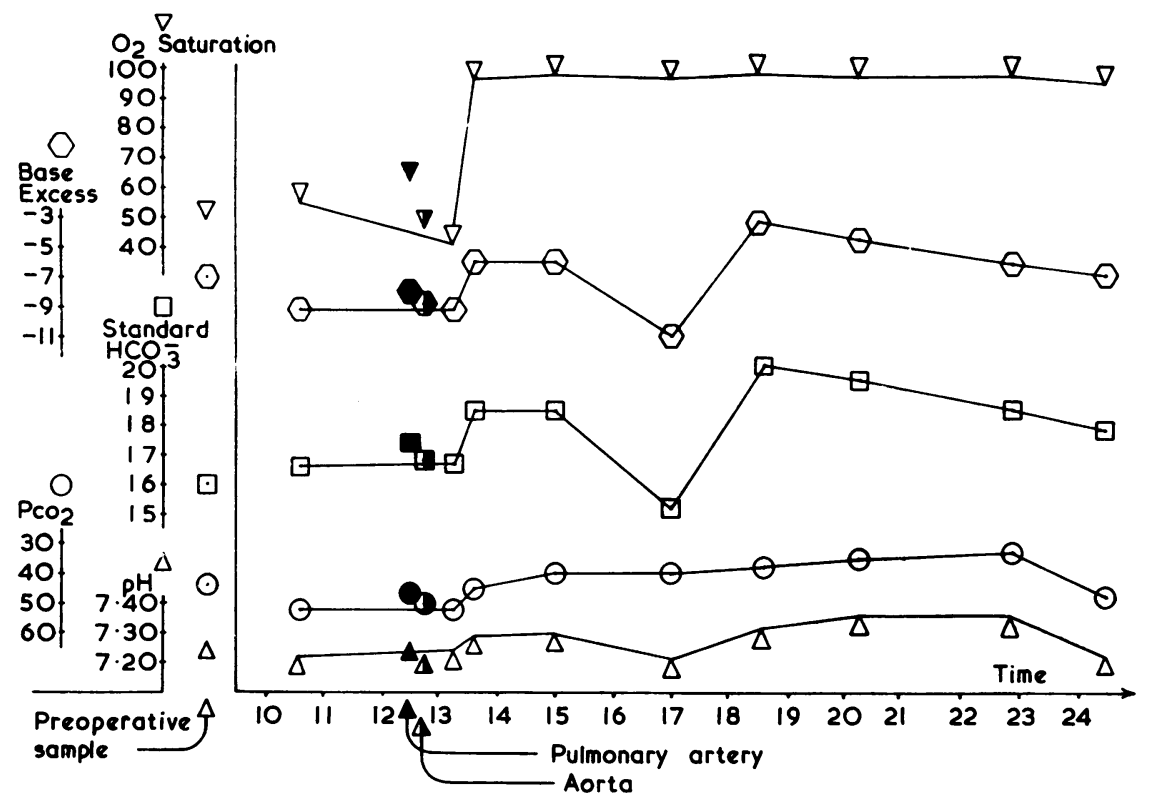

FIG. 3. Biochemical perfusion data.

Fig. 4 presents electroencephalograms taken at various times during the perfusion. Cortical activity may be seen even towards the end of the perfusion, although there are E.E.G. signs of decreasing cerebral activity.

As previous investigations have shown that the machine is not particularly traumatic to the cellular elements of the blood, we no longer perform routine determinations during perfusion, and this was not done in the present case. However, haemolysis was determined once, after about seven hours' perfusion, and was found to be $292 \mathrm{mg}$. $/ 100 \mathrm{ml}$.

In the Rygg-Kyvsgaard oxygenator defoaming of the blood is performed by a sponge which is anti-foam treated. The anti-foaming ability is not unlimited, and after nine hours' perfusion there was so much foam in the oxygenator that it was felt advisable to replace it by another. The fresh oxygenator was primed with freshly drawn donor blood without any air entering the system. During the exchange procedure cardiac massage was kept up. The interruption lasted for four minutes, and the pupils were contracted during the entire exchange procedure.

After about 12 hours' perfusion there was no improvement in the condition of the heart. It was considered that no further benefit could be obtained from the extracorporeal circulation and it was discontinued. Efforts were made to get the heart going again, but without success, and the patient died within a few minutes.

NECROPSY The circumference of the pulmonary artery was about $9.5 \mathrm{~cm}$. and that of the aorta $6 \mathrm{~cm}$. The right ventricle easily admitted an index finger through the pulmonary ostium, whereas the left ventricle only admitted a little finger through the aorta. When the right ventricle was opened the prosthesis was found to be in a fairly good position, but there was a rupture the width of a pencil in the suture at the right limit of the aortic ring. There was copious foamy sanguineous fluid in the air passages. Gross inspection showed no furtner abnormalities. Microscopic examination of the myocardium and liver revealed no abnormality. The lungs were oedematous and showed in several places red cells in the alveoli. In the kidney there was vacuolation of several glomeruli, presumably representing fat emboli or silicone emboli.

The brain weighed $1,250 \mathrm{~g}$. after fixation. There were no gross abnormalities apart from oedema of the leptomeninges and slight compression of the ventricular system. Histological examination of a total of 15 sections from various parts of the cortex with underlying white matter, basal ganglia, brain stem, and cerebellum revealed moderate swelling and chromophobia of the ganglion cells in the cerebral cortex. The cerebellum showed in the stratum granulosum moderate but definite anoxic changes manifesting themselves by clumping and pyknosis of the nuclei as well as intercellular oedema. In addition, there was loss of some Purkinje cells, while others were swollen and chromophobic. Sudan-stained frozen sections did not contain fat emboli, and on the whole there was no abnormal sudanophllic substance in any site. There were no irreparable changes in the cerebrum, whereas the cerebellar changes must be considered irreversible and due to anoxia.

\section{DISCUSSION}

The interesting feature of this case of extracorporeal circulation is whether the patient could have survived if his cardiac condition had made it possible to tide him over the operation. 


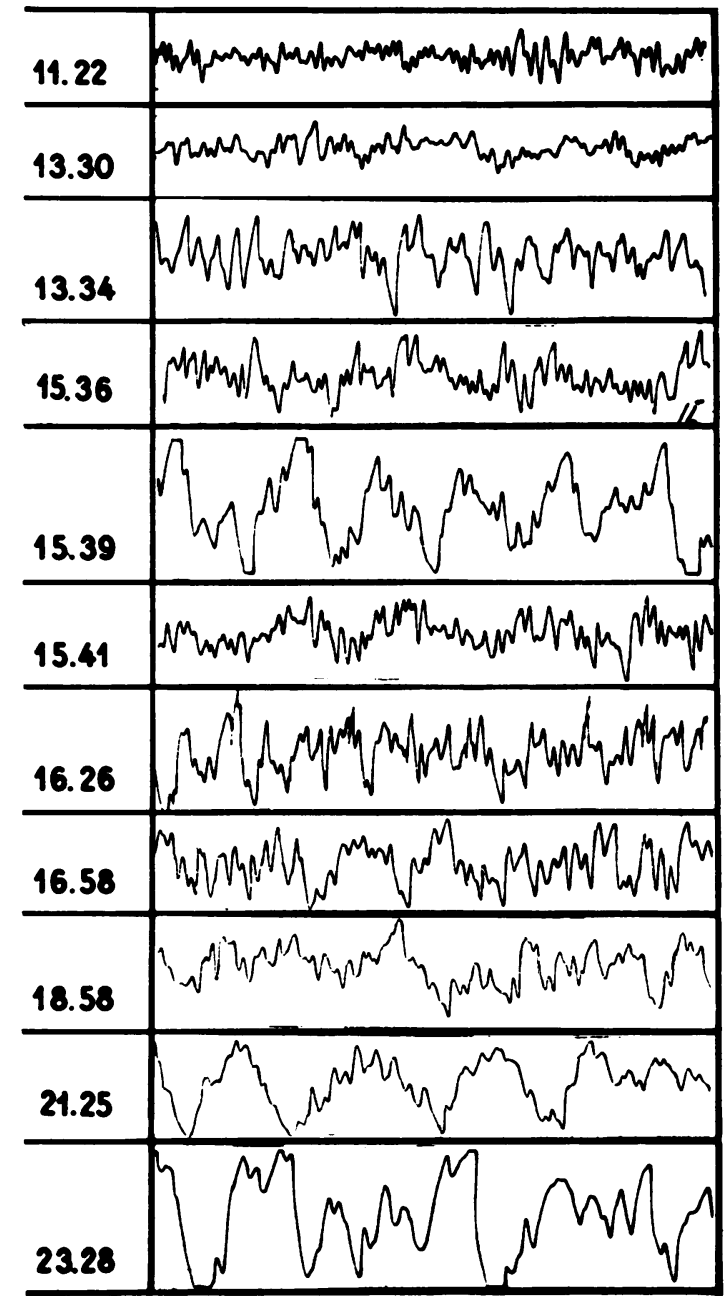

FIG. 4. E.E.G. changes during the perfusion. $11: 22$ Before cardio-pulmonary by-pass; nitrous oxide. 13:30 Start of cardio-pulmonary by-pass. 13:34 Total cardio-pulmonary by-pass; 50 mg. barbiturate. 15:36 Cardio-pulmonary bypass at end of cardiotomy. 15:39 Temporary interruption of cardio-pulmonary by-pass. Severe hypotension. 15:41 Cardio-pulmonary by-pass re-started. 16:26 Temporary interruption of by-pass. Hypotension. 16:58 Cardiopulmonary by-pass after period of severe hypotension. Halothane. 18:58 After $100 \mathrm{mg}$. barbiturate. Halothane just discontinued. $21: 25$ After period of venous hypertension. 23 :28 One hour after exchange of oxygenator.

The oxygen saturation of the blood as well as the flow showed that the oxygen supply ought to have been adequate to the very last. On the other hand, the falling standard bicarbonate and base excess values indicated that the flow through the different parts of the body had been unequal, blood from areas with deficient flow mixing with blood from areas with a sufficient flow, thus giving rise to an increasing acidity of the blood.

As already mentioned, the E.E.G. showed cortical activity right up to the discontinuation of the extracorporeal circulation. This accords with the postmortem findings in the brain, there being no anoxic damage in the cerebrum but only in the cerebellum, which is the most sensitive part of the brain in this respect. The changes would have given rise to symptoms which a child should have been able to tolerate. Some of the E.E.G. changes were due to the fact that the patient's tolerance of the periods of hypotension was reduced at the high temperature. The reason why such a high temperature was maintained was the wish to create better conditions for cardiac restitution.

Microscopic examination of other organs revealed that neither the myocardium nor the liver were damaged by anoxia. There was also no anoxic damage in the kidneys. The microscopic renal changes suggested silicone emboli; but no similar changes were present in the brain. In animal experiments, after administering silicone by spray directly into the blood, silicone emboli have invariably been found in the brain. Indeed there is no reason to believe that silicone emboli were present in the body as no silicone was supplied to the machine during the perfusion. The haemolysis at the end of seven hours' perfusion, $292 \mathrm{mg} . / 100 \mathrm{ml}$., cannot be taken to be excessively high. At least, a value of this order of magnitude is of no pathological significance.

The cellular elements of the blood and the coagulation factors were not investigated. There was no abnormal post-operative bleeding.

\section{SUMMARY}

Extracorporeal circulation was kept up for 12 hours in a patient with transposition of the great vessels and ventricular septal defect. The extracorporeal circulation was performed by the Rygg-Kyvsgaard heart-lung machine. The outcome was fatal. Electroencephalograms showed cortical activity to the very end, and examination of the brain post mortem revealed definite irreparable changes only in the cerebellum. The biochemical changes during the extracorporeal circulation are illustrated graphically.

This work was aided by grants from the Danish State Research Foundation and the P. Carl Petersen Foundation.

\section{REFERENCES}

Andersen, M. N. (1961). J. thorac. cardiovasc. Surg., 41, 244.

Arnfred, E., Rygg, I. H., Frederiksen, T., Engell, H. C., Poulsen, Thue, and Rosen, J. (1961). Thorax, 16, 309.

Frederiksen, T., Rygg, I. H., and Therkelsen, F. (1962). Thoraxchirurgie, 9, 591. 